\title{
SZ effect from Dark Matter annihilation
}

\author{
S. Colafrancesco \\ INAF - Osservatorio Astronomico di Roma via Frascati 33, 00040 Monteporzio, Italy \\ e-mail: cola@mporzio.astro.it \\ Received 8 April 2004 / Accepted 15 May 2004

\begin{abstract}
We derive in this Letter the SZ effect induced by the secondary electrons produced in the annihilation of Weakly Interacting Massive Particles (assumed here to be neutralinos) in gravitationally bound structures dominated by Cold Dark Matter (CDM). We show that the DM induced SZ effect has a specific spectral shape and an amplitude which increases for decreasing neutralino mass $M_{\chi}$. The available SZ data on the Coma cluster set an upper limit on the quantity $\langle\sigma V\rangle_{\mathrm{A}} n_{\chi}^{2}$ which can be combined with the WMAP constraints on $\Omega_{\mathrm{m}} h^{2}$ to restrict the available neutralino models in the $\langle\sigma V\rangle_{\mathrm{A}}-M_{\chi}$ plane. We delineate various potential applications of this method to constraint the physical properties of the Dark Matter particles from the study of galaxy clusters and dwarf spheroidal galaxies in the light of the next coming high-sensitivity SZ experiments.
\end{abstract}

Key words. cosmology - dark matter - galaxies: clusters - cosmic microwave background

\section{Introduction}

Dark Matter (DM) annihilation in the halo of galaxies and galaxy clusters have relevant astrophysical implications. In fact, if DM is constituted by weakly interacting massive particles (WIMPs), their annihilation can produce direct and indirect signals such as observable fluxes of positrons (e.g., Silk \& Srednicki 1984; Kamionkowski \& Turner 1991; Baltz \& Edsjo 1999) antiprotons (e.g., Bottino et al. 1998), gamma rays (e.g., Chardonnet et al. 1995; Colafrancesco \& Mele 2001), neutrinos (e.g., Gondolo \& Silk 1999; Hooper \& Silk 2004), radio emission (e.g., Colafrancesco \& Mele 2001), heating of the hot intra-cluster gas (Totani 2004; Colafrancesco 2004). We do not have yet however, at present, a definite detection of these emission features originating from DM annihilation.

As an alternative strategy, we explore here the consequences of the Compton scattering between the secondary electrons produced from the WIMP anihilation in massive DM halos, like galaxy clusters, and the CMB photon field.

Galaxy clusters are gravitationally dominated by Cold Dark Matter for which the leading candidate is the lightest supersymmetric (SUSY) particle, plausibly the neutralino $\chi$. Experimental and theoretical considerations for having a cosmologically relevant neutralino DM lead to bound its mass $M_{\chi}$ in the range between a few $\mathrm{GeV}$ to a few hundreds of $\mathrm{GeV}$ (e.g., Bottino et al. 2003; Belanger 2003). The decays of neutralino annihilation products (fermions, bosons, etc.) yield, among other particles, energetic electrons and positrons up to energies comparable to the neutralino mass. Here we notice that these energetic electrons and positrons (hereafter we will refer to these particles as electrons because their distinction is not essential for our purpouses) can interact with the CMB photons and up-scatter them to higher frequencies producing a peculiar Sunyaev-Zel'dovich (1972, 1980, hereafter SZ) effect with specific spectral and spatial features. In this Letter, we will describe the specific feature of the SZ effect produced by DM annihilation, $\mathrm{SZ}_{\mathrm{DM}}$, in galaxy clusters and we will discuss the possibility to disentangle such specific $\mathrm{SZ}_{\mathrm{DM}}$ effect from the other sources of SZ effect which are present in the same structures. We will finally discuss the future experimental prospects for the detection of the $\mathrm{SZ}_{\mathrm{DM}}$ effect in $\mathrm{DM}$ halos. The relevant physical quantities are calculated using $H_{0}=70 \mathrm{~km} \mathrm{~s}^{-1} \mathrm{Mpc}^{-1}$ and a flat, vacuum-dominated $\operatorname{CDM}\left(\Omega_{\mathrm{m}}=0.3, \Omega_{\Lambda}=0.7\right)$ cosmological model.

\section{The SZ effect from DM annihilation}

The calculation of the secondary electron spectrum from $\chi \chi$ annihilation in galaxy clusters has been already presented in details by Colafrancesco \& Mele (2001) and here we will only recall the relevant steps which are necessary for the present purpouses.

\subsection{Neutralino annihilation in galaxy clusters}

Following Colafrancesco \& Mele (2001) we assume a spherical, uniform top-hat model for the collapse of a cluster with an average DM density $\bar{\rho}=\Delta\left(\Omega_{0}, z\right) \rho_{\mathrm{b}}\left(1-f_{\mathrm{g}}\right)$ where the background density is $\rho_{\mathrm{b}}=\Omega_{\mathrm{m}} \rho_{\mathrm{c}}$ and $\Delta\left(\Omega_{0}, z\right)$ is the nonlinear density contrast of the halo which virializes at redshift $z$. Here $f_{\mathrm{g}}$ is the gas mass fraction. Redistributing the total mass, $M=\frac{4 \pi}{3} \bar{\rho} R_{\mathrm{p}}^{3}$, found within the radius $R_{\mathrm{p}}=p r_{\mathrm{c}}$ (expressed 
in terms of the cluster core radius $r_{\mathrm{c}}$ ), according to a density profile $\rho(r)=\rho_{0} g(r)$, we find that the central total density is $\rho_{0}=\frac{\bar{\rho}}{3} \frac{R_{\mathrm{p}}^{3}}{I\left(R_{\mathrm{p}}\right)}$, where $I\left(R_{\mathrm{p}}\right)=\int_{0}^{R_{\mathrm{p}}} \mathrm{d} r r^{2} g(r)$. In our phenomenological approach, we consider mainly the case a central cusp profile, as indicated by the available $N$-body simulations. The central cusp model described by a profile:

$g(r)=\left(\frac{r}{r_{\mathrm{c}}}\right)^{-\eta}\left(1+\frac{r}{r_{\mathrm{c}}}\right)^{\eta-\xi}$

gives a central density $\rho_{0}=\frac{\bar{\rho}}{3} \frac{p^{3}}{I(p, \eta, \xi)}$, where $I(p, \eta, \xi)=$ $\int_{0}^{p} \mathrm{~d} x x^{2-\eta}(1+x)^{\eta-\xi}$. With $\eta=1$ and $\xi=3$, the central cusp model corresponds to the "universal density profile" which Navarro et al. (1997) showed to be a good description of cluster DM halos in $N$-body simulations of CDM hierarchical clustering. Assuming that the DM density scales like the total cluster density, the central DM number density is:

$n_{\chi, 0}=\frac{\bar{n}}{3} \frac{p^{3}}{I}$

where

$\bar{n} \approx 4.2 \times 10^{-5} \mathrm{~cm}^{-3} \Omega_{\chi} h^{2}\left[\frac{M_{\chi}}{100 \mathrm{GeV}}\right]^{-1}\left[\frac{\Delta\left(\Omega_{0}, z\right)}{400}\right]$.

The $\chi$ annihilation rate in a DM halo is $R=n_{\chi}(r)\langle\sigma V\rangle_{\mathrm{A}}$, where $n_{\chi}(r)=n_{\chi, 0} g(r)$ is the neutralino number density and $\langle\sigma V\rangle_{\mathrm{A}}$ is the $\chi \chi$ annihilation cross section averaged over a thermal velocity distribution at freeze-out temperature. Although the $\chi \chi$ annihilation cross section is a non-trivial function of the mass and physical composition of the neutralino, to our purpose it suffices to recall that the $\chi$ relic density is approximately given by:

$\Omega_{\chi} h^{2} \simeq \frac{3 \times 10^{-27} \mathrm{~cm}^{3} \mathrm{~s}^{-1}}{\langle\sigma V\rangle_{\mathrm{A}}}$

(Jungman et al. 1996). Hence, for the $\chi \chi$ annihilation, we shall assume a total cross section of $\langle\sigma V\rangle_{\mathrm{A}} \approx 2.6 \times 10^{-26} \mathrm{~cm}^{3} \mathrm{~s}^{-1}$ to be consistent with the value $\Omega_{\mathrm{m}} h^{2} \sim 0.116$ derived from WMAP (Bennett et al. 2003). For values of $\Omega_{\mathrm{m}} h^{2}$ in the range $0.085-0.152$, the annihilation cross section is fixed to within a factor less than three. Detailed studies of the relic neutralino annihilation (Edsjo 1997) show that the above value is well inside the allowed range predicted in supersymmetric theories for a wide choice of masses and physical compositions of neutralinos that can be relevant as CDM candidates. Enhancing (suppressing) the $\chi$ annihilation rate will have on our results the simple effect of rescaling the final electron spectra by the same enhancement (suppression) factor.

Neutralinos which annihilate inside a DM halo produce quarks, leptons, vector bosons and Higgs bosons, depending on their mass and physical composition. Electrons are then produced from the decay of the final heavy fermions and bosons (monochromatic electrons, with energy about $M_{\chi}$, coming from the direct channel $\chi \chi \rightarrow e e$, are in general much suppressed, Turner \& Wilczek 1990). The different composition of the $\chi \chi$ annihilation final state will in general affect the form of the final electron spectrum. Analytical expressions for the $\mathrm{e}^{ \pm}$ spectrum has been given by Colafrancesco \& Mele (2001) and we refer to this paper for further details.
The time evolution of the electron spectrum is given by the transport equation:

$$
\frac{\partial n_{\mathrm{e}}(E, r)}{\partial t}-\frac{\partial}{\partial E}\left[n_{\mathrm{e}}(E, r) b(E)\right]=Q_{\mathrm{e}}(E, r)
$$

where $n_{\mathrm{e}}(E, r)$ is the equilibrium spectrum at distance $r$ from the cluster center for the electrons with energy $E$. The source electron spectrum, $Q_{\mathrm{e}}(E, r)$, rapidly reaches its equilibrium configuration mainly due to synchrotron and Inverse Compton Scattering losses at energies $E \gtrsim 150 \mathrm{MeV}$ and to Coulomb losses at smaller energies (e.g., Colafrancesco \& Mele 2001). Since these energy losses are efficient in the cluster atmosphere and DM annihilation continuously refills the electron spectrum, the population of high-energy electrons can be described by a stationary transport equation

$$
-\frac{\partial}{\partial E}\left[n_{\mathrm{e}}(E, r) b_{\mathrm{e}}(E)\right]=Q_{\mathrm{e}}(E, r)
$$

from which the equilibrium spectrum can be calculated. Here, the function $b_{\mathrm{e}}(E)$ gives the energy loss per unit time at energy $E$

$b_{\mathrm{e}}(E)=b_{0}\left(B_{\mu}\right)\left(\frac{E}{\mathrm{GeV}}\right)^{2}+b_{\mathrm{Coul}}$,

where $b_{0}\left(B_{\mu}\right)=\left(2.5 \times 10^{-17}+2.54 \times 10^{-18} B_{\mu}^{2}\right)$ and $b_{\text {Coul }}=$ $7 \times 10^{-16}\left[n(r) / 1 \mathrm{~cm}^{-3}\right]$, if $b_{\mathrm{e}}$ is given in units of $\mathrm{GeV} / \mathrm{s}$. The source spectra we derived (see Fig. 1 in Colafrancesco \& Mele 2001) arise from an analytic approximation of the exact shape of the electron spectrum that tries to cope with the details of the quarks and leptons decays and of the hadronization of the decay products. Detailed electron spectra can also be obtained by using state-of-the-art Monte Carlo simulations, although the analytical approximations used here can resume the relevant aspects of more detailed studies.

\subsection{The $S Z$ effect from $\chi-\chi$ annihilation products}

The generalized expression for the SZ effect which is valid in the Thomson limit for a generic electron population in the relativistic limit and includes also the effects of multiple scatterings and the combination with other electron population in the cluster atmospheres has been derived by Colafrancesco et al. (2003). This approach is the one that should be properly used to calculate the specific $\mathrm{SZ}_{\mathrm{DM}}$ effect induced by the secondary electrons produced by $\chi \chi$ annihilation. Here we do not repeat the description of the analytical technique and we refer to the general analysis described in Colafrancesco et al. (2003). According to these results, the DM induced spectral distortion can be written as

$\Delta I_{\mathrm{DM}}(x)=2 \frac{\left(k_{\mathrm{B}} T_{0}\right)^{3}}{(h c)^{2}} y_{\mathrm{DM}} \tilde{g}(x)$,

where $T_{0}$ is the $\mathrm{CMB}$ temperature and the Comptonization parameter $y_{\mathrm{DM}}$ is given by

$y_{\mathrm{DM}}=\frac{\sigma_{T}}{m_{\mathrm{e}} c^{2}} \int P_{\mathrm{DM}} \mathrm{d} \ell$ 
in terms of the pressure $P_{\mathrm{DM}}$ contributed by the secondary electrons produced by neutralino annihilation. The quantity $y_{\mathrm{DM}} \propto\langle\sigma V\rangle_{\mathrm{A}} n_{\chi}^{2}$ and scales as $\propto\langle\sigma V\rangle_{\mathrm{A}} M_{\chi}^{-2}$, providing an increasing pressure $P_{\mathrm{DM}}$ and optical depth $\tau_{\mathrm{DM}}=\sigma_{T} \int \mathrm{d} \ell n_{\mathrm{e}}$ for decreasing values of the neutralino mass $M_{\chi}$. The function $\tilde{g}(x)$, with $x \equiv h v / k_{\mathrm{B}} T_{0}$, for the DM produced secondary electron population can be written as

$\tilde{g}(x)=\frac{m_{\mathrm{e}} c^{2}}{\left\langle k_{\mathrm{B}} T_{\mathrm{e}}\right\rangle}\left\{\frac{1}{\tau}\left[\int_{-\infty}^{+\infty} i_{0}\left(x e^{-s}\right) P(s) \mathrm{d} s-i_{0}(x)\right]\right\}$

in terms of the photon redistribution function $P(s)$ and of $i_{0}(x)=2\left(k_{\mathrm{B}} T_{0}\right)^{3} /(h c)^{2} \cdot x^{3} /\left(e^{x}-1\right)$, where we defined the quantity

$$
\begin{aligned}
\left\langle k_{\mathrm{B}} T_{\mathrm{e}}\right\rangle & \equiv \frac{\sigma_{\mathrm{T}}}{\tau} \int P \mathrm{~d} \ell=\frac{\int P \mathrm{~d} \ell}{\int n_{\mathrm{e}} \mathrm{d} \ell} \\
& =\int_{0}^{\infty} \mathrm{d} p f_{\mathrm{e}}(p) \frac{1}{3} p v(p) m_{\mathrm{e}} c
\end{aligned}
$$

(see Colafrancesco et al. 2003) which is the analogous of the average temperature for a thermal population (for a thermal electron distribution $\left\langle k_{\mathrm{B}} T_{\mathrm{e}}\right\rangle=k_{\mathrm{B}} T_{\mathrm{e}}$ obtains, in fact). The photon redistribution function $P(s)=\int \mathrm{d} p f_{\mathrm{e}}(p) P_{\mathrm{s}}(s ; p)$ with $s=\ln \left(v^{\prime} / v\right)$, in terms of the CMB photon frequency increase factor $v^{\prime} / v=\frac{4}{3} \gamma^{2}-\frac{1}{3}$, depends on the electron momentum $(p)$ distribution, $f_{\mathrm{e}}(p)$, produced by $\chi \chi$ annihilation.

Figure 1 shows a comparison between the SZ effect obtained for a thermal electron distribution with $k_{\mathrm{B}} T_{\mathrm{e}}=8.2 \mathrm{keV}$ and that evaluated for the $\chi \chi$ annihilation for different values of $M_{\chi}$. A major difference between the two spectral functions is the different position of the zero of the SZ effect which is moved to higher frequencies in the case of the DM produced electrons with respect to the case of the thermal distribution. As a consequence, the $\mathrm{SZ}_{\mathrm{DM}}$ effect appears as a negative contribution to the overall SZ effect at all the frequencies which are relevant for the SZ experiments, $x \sim 0.5-10$. Note that the amplitude of the $\mathrm{SZ}_{\mathrm{DM}}$ effect increases for decreasing values of the neutralino mass since the quantity $y_{\mathrm{DM}} \propto\langle\sigma V\rangle_{\mathrm{A}} n_{\chi}^{2} \sim M_{\chi}^{-2}$ which accounts for the scaling $n_{\chi} \sim M_{\chi}^{-1}$. A further $M_{\chi}^{-1}$ dependence is present in the electron source function from $\chi \chi$ annihilation (see, e.g., Colafrancesco \& Mele 2001, their appendix).

\subsection{Application to galaxy clusters}

The SZ effect in galaxy clusters is dominated by the Compton scattering produced by the hot, thermal intra-cluster gas. However, it has been shown that there are additional sources of SZ effect which might be produced by other electron populations of thermal (warm IC gas, see e.g. Colafrancesco 2004) and non-thermal (relativistic electrons producing, e.g., the cluster radio-halo emission, Colafrancesco et al. 2003; Colafrancesco 2004) origin residing in the cluster atmosphere. The SZ effect unavoidably induced by DM annihilation in the same cluster combines with the previous SZ effects to produce an overall SZ effect which, hence, retains a complex information about the physical structure of the cluster atmosphere. We will refer, in the following, to the case of the Coma

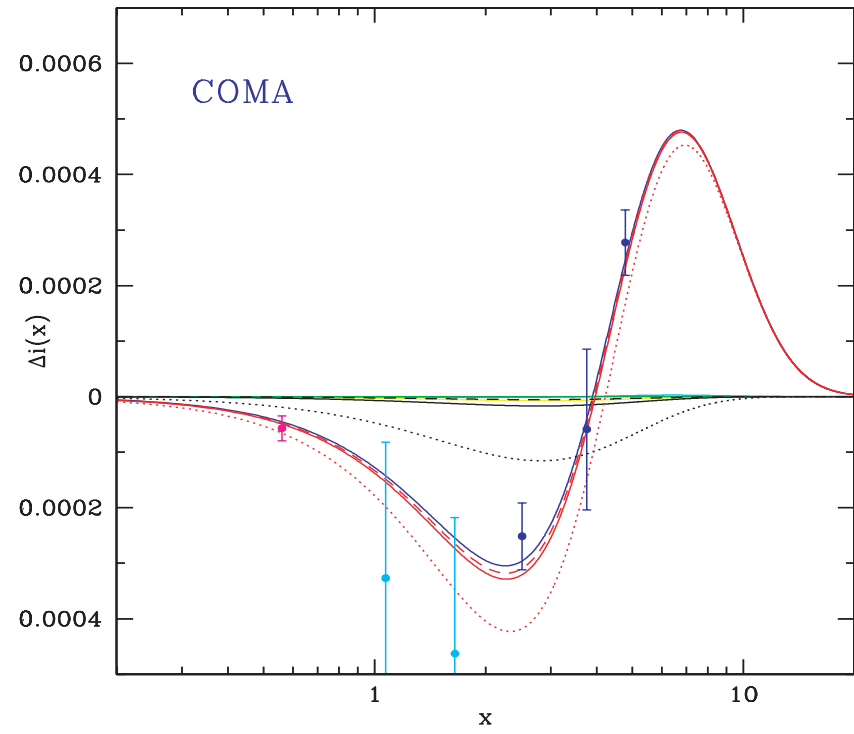

Fig. 1. The overall SZ effect in Coma produced by the combination of various electron populations: thermal hot gas with $k_{\mathrm{B}} T_{\mathrm{e}}=8.2 \mathrm{keV}$ and $\tau=4.9 \times 10^{-3}$ (solid blue curve) which best fits the available SZ data (DePetris et al. 2003); relativistic electrons which best fit the radio-halo spectrum (yellow curve) provide a small additional SZ effect (Colafrancesco et al. 2003); warm gas with $k_{\mathrm{B}} T_{\mathrm{e}} \approx 0.1 \mathrm{keV}$ and $n \approx 10^{-3} \mathrm{~cm}^{-3}$ (cyan curve) provides a small SZ effect due to its low pressure (Colafrancesco 2004); DM produced secondary electrons with $M_{\chi}=10$ (black dotted curve), $20 \mathrm{GeV}$ (black solid curve) and $30 \mathrm{GeV}$ (dashed solid curve). A pure-gaugino $\chi$ reference model is assumed in the computations. The relative overall SZ effect is shown as the dotted, solid and dashed red curves, respectively. A zero peculiar velocity of Coma is assumed consistently with the available limits (Bernardi et al. 2002). SZ data are from OVRO (magenta), WMAP (cyan) and MITO (blue).

cluster for which there are several SZ observations available at different frequencies, from $\sim 30 \mathrm{GHz}$ to $\sim 350 \mathrm{GHz}$. The Coma cluster atmosphere is a complex environment which hosts various electronic populations: a hot, thermal gas with $k_{\mathrm{B}} T_{\mathrm{e}} \approx 8.2 \mathrm{keV}$ producing the bulk of the X-ray emission in the 1-10 keV region; a population of relativistic electrons with a non-thermal spectrum, $n_{\text {rel }} \propto E^{-x}$ with $x \approx 3.5-4$, and energy $E_{\mathrm{e}} \approx 16.4 \mathrm{GeV}_{\mu}^{-1 / 2}(v / \mathrm{GHz})^{1 / 2}$, which are responsible for the radio-halo synchrotron emission (here $B_{\mu}$ is the magnetic field in units of $\mu \mathrm{G}$, see, e.g., Colafrancesco 2004 for a review); a possible warm gas halo with $k_{\mathrm{B}} T_{\mathrm{e}} \approx 0.1 \mathrm{keV}$ claimed to be responsible for the observed soft X-ray/EUV emission excess (e.g., Bonamente et al. 2003, but see also Bowyer et al. 2004 for an alternative explanation); the secondary electrons produced by the DM annihilation under the assumption that DM is mainly constituted by WIMPs. The overall SZ effect from the combination of the different electronic populations in Coma can be evaluated properly in the approach described by Colafrancesco et al. (2003). The total SZ effect for values of $M_{\chi}=10,20$ and $30 \mathrm{GeV}$ is shown in Fig. 1 . To be consistent with the data, the $\mathrm{SZ}_{\mathrm{DM}}$ effect cannot be due to neutralinos with $M_{\chi} \lesssim 20 \mathrm{GeV}$ for the assumed value of $\langle\sigma V\rangle_{\mathrm{A}}$. Since $\mathrm{SZ}_{\mathrm{DM}}$ depends on the quantity $\langle\sigma V\rangle_{\mathrm{A}} n_{\chi}^{2}$, the available SZ data set actually an upper limit in the $\langle\sigma V\rangle_{\mathrm{A}}-M_{\chi}$ plane (see Fig. 2). Models 


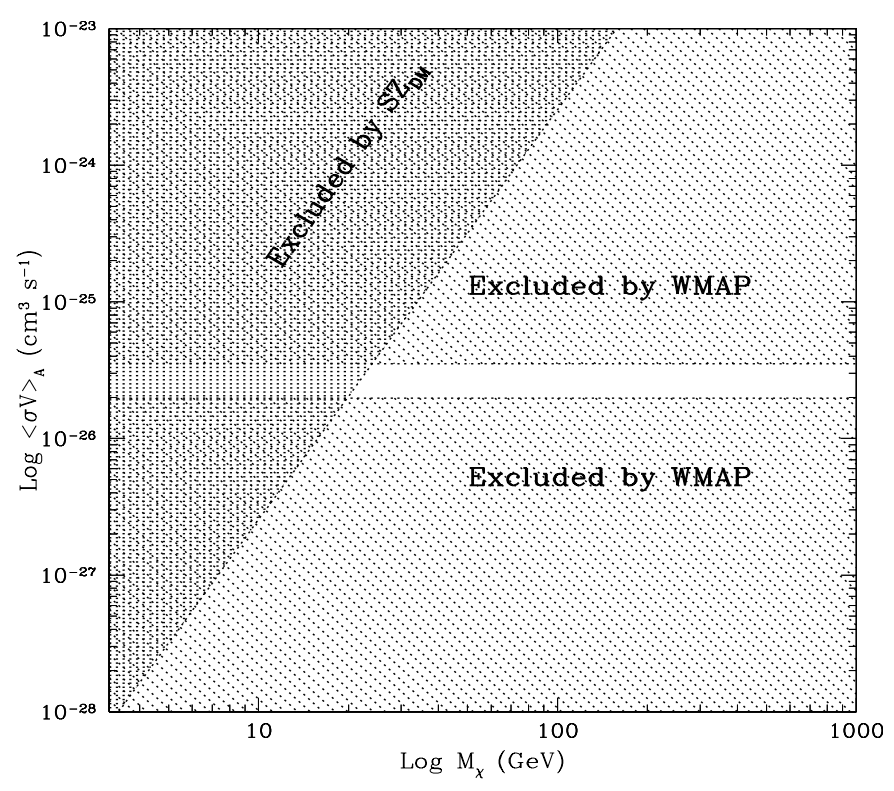

Fig. 2. The constraints on the $\langle\sigma V\rangle_{\mathrm{A}}-M_{\chi}$ plane set by the SZ effect from Coma. The heavily dashed area is excluded by the analysis of the $\mathrm{SZ}_{\mathrm{DM}}$ in Coma. The SZ constraints are combined with the WMAP constraint $0.085 \lesssim \Omega_{\mathrm{m}} h^{2} \lesssim 0.152$ (Bennett et al. 2003) which are translated on the quantity $\langle\sigma V\rangle_{\mathrm{A}}$ through Eq. (4).

with large values of $\langle\sigma V\rangle_{\mathrm{A}}$ and low values of $M_{\chi}$ which are found in the shaded area are excluded by the excess $\mathrm{SZ}_{\mathrm{DM}}$ effect in Coma. The limits on $\langle\sigma V\rangle_{\mathrm{A}}$ set by WMAP restrict further on the available region of the $\langle\sigma V\rangle_{\mathrm{A}}-M_{\chi}$ plane.

\section{Conclusions and future prospects}

We have shown that the SZ effect induced by secondary electrons produced in $\chi \chi$ annihilation is an unavoidable consequence of the presence and of the nature of Dark Matter in large-scale structures. The analysis of the DM induced SZ effect in galaxy clusters provides a complementary probe for the presence and for the nature of DM in cosmic structures. The available SZ observations on the Coma cluster (see Fig. 2) can already set a lower limit to the neutralino mass of $M_{\chi} \gtrsim$ $17-20 \mathrm{GeV}\left(M_{\chi} \gtrsim 13 \mathrm{GeV}\right.$ at $90 \%$ c.l. with the adopted value of $\left.\langle\sigma V\rangle_{\mathrm{A}}\right)$, which are consistent with the limits set by accelerators (e.g., Belanger et al. 2003). The $\mathrm{SZ}_{\mathrm{DM}}$ signal does not strongly depend on the assumed DM density profile at intermediate angular distances from the cluster center and on the DM clumpiness since $y_{\mathrm{DM}}$ is the integral of the total $P_{\mathrm{DM}}$ along the line of sight. The presence of a substantial $\mathrm{SZ}_{\mathrm{DM}}$ effect is likely to dominate the overall SZ signal at frequencies $x \gtrsim 3.8-4.5$ providing a negative total SZ effect (see Fig. 1). It is, however, necessary to stress that in such frequency range there are other possible contributions to the $\mathrm{SZ}$ effect, like the kinematic effect and the non-thermal effect which could provide additional biases (see, e.g., Colafrancesco et al. 2003). Nonetheless, the peculiar spectral shape of the $\mathrm{SZ}_{\mathrm{DM}}$ effect is quite different from that of the kinematic SZ effect and of the thermal SZ effect and this result allows to disentangle it from the overall SZ signal. An appropriate multifrequency analysis of the overall SZ effect based on observations performed on a wide spectral range (from the radio to the sub-mm region) is required, in principle, to separate the various SZ contributions and to provide an estimate of the DM induced SZ effect. In fact, simultaneous $\mathrm{SZ}$ observations at $\sim 150 \mathrm{GHz}$ (where the $\mathrm{SZ}_{\mathrm{DM}}$ deepens the minimum with respect to the dominant thermal SZ effect), at $\sim 220 \mathrm{GHz}$ (where the $\mathrm{SZ}_{\mathrm{DM}}$ dominates the overall $\mathrm{SZ}$ effect and produces a negative signal instead of the expected $\approx$ null signal) and at $\gtrsim 250 \mathrm{GHz}$ (where the still negative $\mathrm{SZ}_{\mathrm{DM}}$ decreases the overall SZ effect with respect to the dominant thermal SZ effect) coupled with X-ray observations which determine the gas distribution within the cluster (and hence the associated dominant thermal $\mathrm{SZ}$ effect) can separate the $\mathrm{SZ}_{\mathrm{DM}}$ from the overall SZ signal, and consequently, set constraints on the neutralino mass. Observations of the radio-halo emission in the cluster can provide an estimate of the cosmic-ray electron population and consequently an estimate of the associated non-thermal SZ effect (which is usually quite small and with a different spectral shape at high frequencies, see e.g., Colafrancesco et al. 2003). The high sensitivity planned for the future SZ experiments, especially at frequencies $x \approx 2.5$ and $x \gtrsim 3.8$, where the $\mathrm{SZ}_{\mathrm{DM}}$ more clearly manifests itself, can provide much stringent limits to the additional SZ effect induced by DM annihilation. In this context, the next coming PLANCK-HFI experiment has enough sensitivity to probe in details the contributions of various SZ effects in the frequency range $x \approx 2-5$. Because the amplitude of the $\mathrm{SZ}_{\mathrm{DM}}$ effect increases with decreasing values of $M_{\chi}$, the high-sensitivity SZ experiments have - hence - the possibility to set reliable constraints to the nature, amount and spatial distribution of DM in galaxy clusters. We will present elsewhere (Colafrancesco 2004 , in preparation) a more extended analysis of DM models in the context of SZ observations.

An exciting possibility in this context could be offered by nearby (with small or zero peculiar velocity) systems which are gravitationally dominated by Dark Matter, which contain little or no gas (in either hot or warm forms) and show absence of non-thermal phenomena connected with the presence of cosmic rays. In such ideal DM systems, the major source of SZ effect would be just the one due to the annihilation of the WIMPs. Systems which could be assimilable to the ideal "pure" DM halos are dwarf spheroidal galaxies and/or low surface brightness galaxies. These systems seem to be ideal sites for studying the DM annihilation indirect signals which reveal themselves in a variety of astrophysical phenomena, whose main imprint is the specific gamma-ray emission (see, e.g., Evans et al. 2003). In such a context, the possible detection of the DM induced SZ effect will provide an important complementary approach which can be studied by more traditional astronomical techniques.

Acknowledgements. The author thanks the Referee for useful comments and P. Marchegiani and S. Profumo for useful discussions.

\section{References}

Baltz, E. A., \& Edsjo, J. 1999, Phys. Rev. D, 59, 2, 15

Belanger, G., et al. 2003, preprint [arXiv: astro-ph/0310037]

Bennett, C. L., Hill, R. S., Hinshaw, G., et al. 2003, ApJS, 148, 175

Bernardi, M., Alonso, M. V., da Costa, L. N., et al. 2002, AJ, 123, 2990 
Bonamente, M., Joy, M. K., \& Lieu, R. 2003, ApJ, 585, 722

Bottino, S., Donato, F., Fornengo, N., \& Salati, P. 1998, Phys. Rev. D, 58,1215

Bottino, S., Donato, F., Fornengo, N., \& Scopel, S. 2003, Phys. Rev. D, 68, 043506

Bowyer, S., et al. 2004, ApJ, submitted, preprint [arXiv: astro-ph/0403181]

Chardonnet, P., Salati, P., Silk, J., Grenier, I., \& Smoot, G. 1995, ApJ, 454,754

Colafrancesco, S., \& Mele, B. 2001, ApJ, 562, 24

Colafrancesco, S. 2004, J. Nucl. Phys., in press

Colafrancesco, S., Marchegiani, P., \& Palladino, E. 2003, A\&A, 397,27

Colafrancesco, S. 2004, in Soft X-ray excess emission from clusters of galaxies, ed. R. Lieu, \& J. Mittaz, 137, 147 [arXiv: astro-ph/0403404]

De Petris, M., D’ Alba, L., Lamagna, L., et al. 2002, ApJ, 574, L119
Evans, N. W., Ferrer, F., \& Sarkar, S. 2003, Phys. Rev. D, in press, preprint [arXiv: astro-ph/0311145]

Gondolo, P., \& Silk, J. 1999, Phys. Rev. Lett., 83, 1719

Hooper, D., \& Silk, J. 2004, New J. Phys., 6, 023

Itoh, N., Kohyama, Y., \& Nozawa, S. 1998, ApJ, 502, 7

Jungman, G., Kamionkowski, M., \& Griest, K. 1996, Phys. Rep., 267, 195

Kamionkowski, M., \& Turner, M. S. 1991, Phys. Rev. D, 43, 1774

Navarro, J., Frenk, C., \& White, S. D. M. 1997, ApJ, 490, 493

Silk, J., \& Srednicki, M. 1984, Phys. Rev. Lett., 53, 624

Sunyaev, R. A., \& Zel'dovich, Ya. B. 1972, Comments Astrophys. Space Sci., 4, 173

Sunyaev, R. A., \& Zel'dovich, Ya. B. 1980, ARA\&A, 18, 537

Totani, T. 2003, Phys. Rev. Lett., in press, preprint [arXiv: astro-ph/0401140]

Turner, M. S., \& Wilczek, F. 1990, Phys. Rev. D, 42, 1001 